endorsed in World Health Assembly (WHA) have the potential to better support national and subnational health systems guidance and policy development processes by including information about the contextual factors that can shape decisions about health systems.

Objectives To assess the extent to which WHO health systems guidance and the WHA technical documents include information about how to address a health system problem and how the health system arrangements and political system features can influence decision-making.

Methods We reviewed all WHO guidance published since 2008 to 2012 and WHA resolutions published from 2005 to 2012 and included those with a focus on health systems. Two reviewers independently screened and applied the selection criteria to all the documents and extracted the information following pre-established data-extraction forms.

Results 13 out of 78 WHO guidance and 14 technical documents out of 207 WHA resolutions had a health system's focus. Six WHO guidance and 12 WHA documents included information about how to address a health system problem. All WHO guidance included information about delivery arrangements but only three discussed financial arrangements. Two WHO guidance and five WHA documents discussed key features of political systems.

Discussions The inclusion of contextual factors, mainly financial arrangements of health systems and political systems features was infrequent among the reviewed documents.

Implications for Guideline Developers/Users It is necessary to understand better how to integrate these contextual factors in the process of global guidance development.

\section{P210 CHANGES IN PERFORMANCE OF THE ADAPTATION METHODOLOGY USED FOR GUIDELINE DEVELOPMENT FROM 2002 TO 2012}

C Khan, S Schwarz, S Conrad, K Koltermann, A Dippmann, G Ollenschlaeger. Agency for Quality in Medicine (AQuMed), Berlin, Germany

\section{0:1136/bmjqs-2013-002293.208}

Background Guideline adaptation has always been the methodological basis of the German National Disease Management Guideline (NDMG) Programme. Although it allows highly efficient and resource-saving workflows, the NDMG development process becomes an increasingly time-consuming and resourceintensive challenge.

Objective The objective of the present project was to analyse the performance of the adaptation methods used for the NDMG guidelines development to identify the critical issues causing an extensive effort and to find possible coping solutions.

Methods We reviewed each NDMG method report from the beginning of the programme in 2002 to 2012. We extracted and evaluated the duration of the process, the number of source guidelines used and number and themes of additional literature searches over the course of years also in comparison of the first and second editions.

Results One of the 14 NDMGs was excluded because of missing adaptation method during the updating process. The comparison of first half of the programme between 2002 to 2007 and second half from 2007 to 2012 revealed an average duration of the development process of 22,7 versus 49,9 months. Until 2007 4,5 and after 20073,7 source guidelines were used on average. The number of topics of systematic literature searches has been rising over the years. On average 5 additional searches were conducted in the last 5 years. $42 \%$ of all topics covered pharmacotherapy.

Discussion The adaptation methodology is still very useful and efficient. However, prioritisation and consensus processes and strict orientation on the selected source guidelines need to be optimised to reduce the increasing effort.

\section{P211 SYSTEMATIC REVIEW AND QUALITY APPRAISAL OF CLINICAL PRACTICE GUIDELINES FOR THE TREATMENT OF PATIENTS WITH ACUTE CORONARY SYNDROME}

L Lugo, N Acosta, J Senior. University of Antioquia, Medellin, Colombia

\section{0:1136/bmjqs-2013-002293.209}

Background In Colombia, ischemic heart disease was the leading cause of death in people over 55 years of age. In the next five years, the Colombian population over 45 years of age will triple leading to an increase in the incidence and prevalence of atherosclerotic heart disease. Under this scenario, the Colombian Ministry of Health commissioned us the development of a clinical practice guideline (CPG) for the comprehensive treatment of patients with acute coronary syndrome (ACS). Therefore, as part of this process we conducted a systematic review and a quality appraisal of the published CPGs for this condition.

Methods By undertaking a systematic search of multiple databases, reviewing the reference lists of included studies and the input of experts, we identified CPG for ACS with and without ST elevation, published from 2005 to 2011. We assessed the quality of each guideline using the AGREE II instrument.

Results We identified $121 \mathrm{CPG}$ and assessed the quality of 28 that met the inclusion criteria. The overall average for the 28 guides in all domains was: clarity of presentation 91\%, reach and objectives $78 \%$, methodological rigour $72 \%$, stakeholder involvement $62 \%$, editorial independence $72 \%$, and applicability $44 \%$. 11 of 28 guidelines had a low methodological rigour (score less than 60\%).

Discussion The applicability of the guideline, that is, to provide clear identification of tools to implement the recommendations of the guideline, should be strengthened in the CPG for SCA.

\section{P212 REVIEW OF SYSTEMATIC REVIEWS RELATED TO CLINICAL GUIDELINES IMPLEMENTATION}

${ }^{1} \mathrm{D}$ Geba, ${ }^{2} \mathrm{~W}$ Chan, ${ }^{1} \mathrm{M}$ Moreno, ${ }^{1} \mathrm{~T}$ Pearson. ' University of Rochester, Rochester, USA; ${ }^{2}$ Kaiser Permanente, Portland, USA

\section{0:1136/bmjqs-2013-002293.210}

Background Clinical guidelines should be implemented using evidence-based implementation strategies. However, guideline development programmes rarely allocate resources to perform evidence-based reviews of implementation strategies required to implement their guidelines. A streamlined approach to obtain such summaries of evidence in preparation for development of cardiovascular risk reduction guidelines sponsored by a nationallevel organisation was a review of systematic reviews (SRs) of implementation strategies.

Objectives To explore whether SRs of implementation strategies provide support for the effectiveness of these strategies. Methods $\mathrm{Rx}$ for Change database of the Canadian Agency for Drugs and 
Technologies in Health (CADTH) was selected a priori as data source for this review of systematic reviews. The review was limited to high quality SRs of interventions targeting clinicians.

Results A total of 12 SRs met study inclusion criteria. These SRs suggest that implementation strategies, such as audit and feedback, academic detailing, and educational meetings, are generally effective in improving providers' behaviours, with small to moderate effect sizes.

Discussion This review of SRs provides support for the overall efficacy of guideline implementation strategies, while highlighting the need for further comparative and cost effectiveness research to address gaps in the knowledge identified (e.g., limited information on head-to-head comparisons between strategies, clinical context, and cost of interventions).

Implications for Guideline Developers/Users Guideline developers should include recommendations for guideline implementation in their future guidelines. Making specific recommendations on choosing one implementation strategy over the others should be avoided until further head-to-head comparisons are available.

\section{P217 THE EFFECT OF PRINT OR ONLINE EDUCATIONAL MATERIALS FOR PRIMARY CARE PHYSICIANS: A SYSTEMATIC REVIEW}

${ }^{1,2} \mathrm{~A}$ Grudniewicz, ${ }^{3} \mathrm{R}$ Rodseth, ${ }^{1} \mathrm{R}$ Kealy, ${ }^{1} \mathrm{D}$ Rudoler. 'University of Toronto, Toronto,
Canada; ${ }^{2}$ Li Ka Shing Knowledge Institute, St. Michael's Hospital, Toronto, Canada; ${ }^{3}$ University of KwaZulu Natal, Durban, South Africa

\section{0:1136/bmjqs-2013-002293.211}

Background Print and online materials such as guideline summaries are commonly used to distribute evidence to primary care physicians; they are easy to implement and scale across many primary clinics.

Objectives We sought to determine: 1) if providing primary care physicians with print and online educational materials has an effect on physician behaviour or on patient outcomes, 2) how these materials were developed, and 3) whether design attributes impact outcomes.

Methods We systematically identified studies that reported a print or online educational intervention for primary care physicians. Studies were identified by searching four electronic databases, scanning reference lists, and contacting experts. A subanalysis was conducted to collect data on how these materials were developed and on their use of design principles.

Results Thirty studies met eligibility criteria after full-text screening. Studies targeted physician advice-giving behaviour, diagnostic procedures, prescribing behaviour, change in knowledge, and clinical patient outcomes. Results suggest that print and online materials targeted at primary care physicians have little to no effect on outcomes.

Discussion Print and online educational materials provided to primary care physicians have little effect on physician or patient outcomes. This is concerning as they are a common method of disseminating evidence. Most studies do not describe how interventional materials were developed or whether design principles were applied.

Implications for Guideline Developers/Users Design principles should be considered when developing evidence-based materials and the development processes should be described in order to determine if better designs influence uptake and use of evidence.

\section{P219 ENGAGING CONSUMERS IN THE GUIDELINE DEVELOPMENT PROCESS - THE US PERSPECTIVE}

P Robertson, H Hussey, S Jones. American Academy of Otolaryngology-Head and Neck Surgery Foundation, Alexandria, USA

10:1136/bmjqs-2013-002293.212

Background In the United States, there have been increasing calls for guideline developers to engage consumers throughout the guideline development process. The Guidelines International Network (G-I-N) and the Institute of Medicine (IOM) have both released guideline development best practices encouraging consumer involvement; ranging from consumer input during the formulation of clinical questions, to serving as a guideline panel member and participating in the review process.

Context Our organisation has been developing evidence based clinical practice guidelines for nearly a decade and has incorporated consumers in the development process for over five years. By including consumers, our guidelines now feature more patient-centred recommendations; establishing a more balanced discussion of patient preferences and improving how we assess benefits and harms.

Description of Best Practice To more readily identify consumers for guideline development, our organisation has built a collaborative relationship with a consumer advocacy alliance. Through this relationship, we have been able to support two consumer advocates as full members on each guideline panel. To assist their participation, we provide education about our guideline development process, and outline the expectations of their involvement throughout the process.

Lessons for Guideline Developers Our experience with consumer engagement can serve as an example for other US guideline developers. Consumers can bring invaluable insight and perspective throughout guideline development and have substantially improved our guidelines. We believe consumer participation will become increasingly important in the coming years, particularly as guideline developers move towards a standardised approach to development.

\section{P222 EVOLUTION OF EVIDENCE GRADING SYSTEMS IN THE AMERICAN ACADEMY OF OPHTHALMOLOGY'S PREFERRED PRACTICE PATTERNS}

N Emptage, F Lum. American Academy of Ophthalmology, San Francisco, USA

\section{0:1136/bmjqs-2013-002293.213}

Background Clinical practice guidelines are an important component of efforts to improve quality of care and rationalise the introduction of new medical technologies. As evidence-based medicine and comparative effectiveness research become more prominent, the rigour and transparency of guideline development is becoming increasingly important.

Context Since 1988, the American Academy of Ophthalmology has published ophthalmic practice guidelines known as Preferred Practice Patterns (PPPs). Over time, the Academy has introduced increasingly rigorous processes for grading the evidence underpinning the PPPs.

Description of Best Practice Prior to 2000, the PPPs were effectively consensus-based, with no formal processes for identifying or synthesising evidence, and no system for grading evidence quality. In 2000, a three-level system was introduced to denote the quality of the evidence supporting PPP recommendations, 